\title{
Analytical Solution of Substrate Concentration in the Biosensor Response
}

\author{
Seyed Ali Madani Tonekaboni ${ }^{1}$, Ali Shahbazi Mastan Abad ${ }^{2}$, Amin Afshari ${ }^{2}$, Ali Khalilzadeh', \\ Shahab Karimi ${ }^{2}$, Mitra Shabanisamghabady ${ }^{2}$ \\ ${ }^{1}$ School of Mechanical Engineering, University of Waterloo, Waterloo, Canada \\ ${ }^{2}$ School of Mechanical Engineering, University of Tehran, Tehran, Iran \\ Email: ali.madani.1368@gmail.com, ali_shahbazi1990@yahoo.com,amin.afshari90@gmail.com, \\ alikhalilzadeh2004@yahoo.com, shahab.karimi.sk@gmail.com,mitrashabani@ymail.com
}

Received September 8, 2013; revised October 8, 2013; accepted October 15, 2013

Copyright (c) 2013 Seyed Ali Madani Tonekaboni et al. This is an open access article distributed under the Creative Commons Attribution License, which permits unrestricted use, distribution, and reproduction in any medium, provided the original work is properly cited.

\begin{abstract}
Homotopy analysis method (HAM) is employed to investigate amperometric biosensor at mixed enzyme kinetics and diffusion limitation. Mathematical modeling of the problem is developed utilizing non-Michaelis-Menten kinetics of the enzymatic reaction. Different results of the problem are obtained for different values of the dimensionless parameters. Accuracy of the obtained results is verified by comparing them with the available actual and simulated ones. It is concluded that the obtained solution can be considered as a promising one to investigate different aspects of the phenomena.
\end{abstract}

Keywords: Homotopy Analysis Method; Amperometric Biosensor; Mathematical Modeling; Non-Michaelis-Menten Kinetics

\section{Introduction}

Biosensors play important roles as components of the transduction mechanisms [1] and can be employed as measurement devices to gauge biologically relevant information such as neural interfaces and oxygen electrodes [2]. Furthermore, they can be used as transducers which translate the biomolecular responses into electrical signals [3]. Biosensors produce signals harmonized to the concentrations of the measured analytes. These devices are used in so many applications like detection of pathogens [4], toxic metabolites (such as mycotoxins [5]), detection of pesticides and river water contaminants such as heavy metal ions [6], etc. The mentioned examples show the importance of biosensors and their applications in different branches of Science and Engineering which reveals the requirement of analysis of these highly demanded instruments. One of the popular and perspective trends of biosensorics is amperometric biosensor [7]. Since they were first introduced by Clark and Lyons in 1962 [8], several studies have investigated different aspects of amperometric biosensors. In principle, they measure the changes of the current of indicator electrode by direct electrochemical oxidation or reduction of the products of the biochemical reaction [9-11]. They are widely used today because of the reliability and high sensitivity for environment, clinical and industrial applications.

Design of biosensors is based on understanding the kinetic characteristics of these devices. Generally, measuring the concentration of substrate inside enzyme membranes is not possible. Hence, various mathematical models of amperometric biosensors have been presented and used as an important tool in order to obtain analytical characteristics of actual biosensors [12,13], such as investigative monolayer membrane model used to study the biochemical treatment of biosensors $[14,15]$. Their mathematical models are based on reaction-diffusion equations including non-linear term that relate to non-Michaelis-Mentenkinetics of the enzymatic reaction [16,17]. Hence, high accurate analytical and numerical methods should be employed to investigate this important nonlinear chemical equation.

Most scientific problems in engineering are inherently nonlinear. Except for a few of them, the majority of nonlinear problems does not have analytical solutions. 
Therefore, the constitutive laws of these problems should be solved using other schemes such as numerical or perturbation methods. In the numerical method, stability and convergence of the solution should be considered so as to avoid divergence or inappropriate results [18]. In the perturbation method, the small parameter is inserted in the equation; thus, finding the small parameter and exerting it into the equation is one of the deficiencies of this method [19]. One of the semi-exact methods for solving nonlinear equation which does not need small/large parameters is Homotopy Analysis Method (HAM), first proposed by Liao [20,21]. Homotopy Analysis Method is now widely used to solve different types of nonlinear problems. Various papers on nonlinear physical and engineering problems $[22,23]$ have proved the validity of HAM. Moreover, recently the application of HAM on medical and chemistry problems has gotten so much attention among researchers. Counting reaction network equilibria [24], reaction-diffusion Brusselator model [25], predicting the lowest energy conformations of proteins [26] and many other examples can be considered as applications of HAM in Chemistry and Medicine. Several auxiliary parameters and functions available in the procedure of HAM need to be chosen so properly for the convergence of the solution. Through practice of auxiliary parameter h, convergence region of the solution is readily adjustable to a wide range of variables.

This paper presents the analytical solution for an amperometric biosensor at mixed enzyme kinetics and diffusion limitation by utilizing HAM as a strong method. Non-Michaelis-Menten kinetics of the enzymatic reaction is used to obtain the constitutive equation of the problem. Different non-dimensional parameters are defined so as to non-dimensionalize the equation. The obtained non-dimensional equation is used to procure $m^{\text {th }}$ order deformation equation as an important step of the procedure of the solution. The $h$-curves are obtained for several cases illustrated in the paper to clarify the convergence region of the solution. In addition, results are obtained to investigate the effects of the variations of each dimensionless parameter of the procured equation. Finally, some of the results are compared with the actual and simulated results available in the literature [27] to verify the accuracy of the method.

\section{Mathematical Modeling}

Spatial dependency of enzyme kinetics on biochemical systems has recently attracted much attention by considering the effect of diffusion in these processes $[16,17]$. The simplest scheme of non Michaelis-Menten kinetics may for instance be described by adding to the Michaelis-Menten scheme (2.1) the relationship of the interacttion of the enzyme substrate complex $(E S)$ with an- other substrate molecule $(S)$ (2.2) followed by the generation of non-active complex $\left(E S_{2}\right)$ as

$$
\begin{gathered}
E+S \leftrightarrow E S \rightarrow E+P \\
E S+S \leftrightarrow E S_{2}
\end{gathered}
$$

The reaction is sometimes said to display MichaelisMenten kinetics in which the relationship between the rate of an enzyme catalyzed reaction and the substrate concentration takes the form

$$
v=\frac{V_{\max }[S]}{K_{M}+[S]}
$$

where $v$ and $V_{\max }$ are the so-called "initial reaction velocity" and maximum velocity respectively.

In addition, $K_{M}$ is known as Michaelis constant for $S . K_{M}$ and $V_{\max }$ are constants at a given temperature and a given enzyme concentration.

The reactions exhibit non-Michaelis-Menten kinetics, in which the kinetic behavior does not obey the Equation (2.3). The velocity function $v$ for the simple reaction process without competitive inhibition is given by Pao [28] and Baronas et al. [27], which is based on the nonMichaelis-Menten hypothesis,

$$
v=\frac{k_{c}[E]_{0}[S]}{K_{M}+[S]+[S]^{2} / K_{i}}=\frac{V_{\max }[S]}{K_{M}+[S]+[S]^{2} / K_{i}}
$$

where the constants $V_{\max }=k_{c}[E]_{0}, K_{M}$ and $K_{i}$ are Michaelis-Menten and inhibition constants respectively. The Equation (2.4) conforms to Equation (2.3) for large values of $K_{i}$ with respect to $K_{M}$. On the basis of Equation (2.4), the rate is maximized by increasing the concentration. It is then said to be inhibited by the substrate. In addition, the constant $K_{i}$ (which has the dimension of a concentration) is called the substrate inhibition constant. For obtaining the rate of change of substrate concentration $S=S(\eta, t)$ at time $t$ and position $\eta \in \Omega$ throughout the domain, the following equation given by Pao [28] is used.

$$
\frac{\partial S}{\partial t}=D_{S} \nabla \cdot(\nabla S)-v(\eta, t)
$$

$D_{S}$ is the substrate diffusion coefficient and $\nabla S$ is the gradient operation. On the basis of non-MichaelisMenten kinetics, Equation (2.5) becomes

$$
\frac{\partial S}{\partial t}=D_{S} \frac{\partial^{2} S}{\partial \eta^{2}}-\frac{K S}{1+S / K_{M}+S^{2} / K_{i} K_{M}}
$$

in which $K=K_{c} E_{0} / K_{M}$. In this article, steady state condition is considered which results in changing Equation (2.6) to the following equation 


$$
D_{S} \frac{\partial^{2} S}{\partial \eta^{2}}-\frac{K S}{1+S / K_{M}+S^{2} / K_{i} K_{M}}=0
$$

Equation (2.7) is changed to the non-dimensional form (Equation (2.8)) [27] using the following non-dimensional parameters

$$
\begin{aligned}
& \frac{\partial^{2} u}{\partial x^{2}}-\frac{K u}{1+\alpha u+\beta u^{2}}=0, \quad 0<u \leq 1 \\
& u=\frac{S}{k s^{\infty}}, x=\frac{\eta}{L}, K=\frac{k L^{2}}{D_{S}}=\phi^{2}, \alpha=\frac{k s^{\infty}}{K_{M}}, \beta=\frac{k s^{\infty}}{K_{i} K_{M}}
\end{aligned}
$$

Equation (2.8) must be solved to satisfy the following boundary conditions which are based on the location of electrodes and diffusion layer in the boundaries of the membarne

$$
\begin{array}{lll}
u=1 & \text { at } & x=1 \\
\frac{\partial u}{\partial x}=0 & \text { at } & x=0
\end{array}
$$

\section{HAM Solution}

In this section the solution procedure of this problem using HAM is discussed. The appropriate form of nonlinear differential Equation (2.8) for the procedure of HAM is presented as follows:

$$
\begin{aligned}
& N[\varphi(x, q)]=\left(1+\alpha \varphi(x, q)+\beta(\varphi(x, q))^{2}\right) \\
& \frac{\partial^{2}}{\partial x^{2}}(\varphi(x, q))-K \varphi(x, q)=0
\end{aligned}
$$

where $i$ is the node number, $N$ is the nonlinear operator, and the function $\varphi_{i}(q)$ is defined as

$$
\begin{aligned}
& \lim _{q \rightarrow 0} \varphi(x, q)=u_{0}(x), \\
& \lim _{q \rightarrow 1} \varphi(x, q)=u(x)
\end{aligned}
$$

where $u(x)$ is the unknown field variable, $q \in(0,1)$ is the embedding parameter, and $u_{0}(x)$ is the initial guess which is employed to meet the requirements of the boundary conditions. In this paper, the $u_{0}[x]=1$ has been chosen which correctly satisfies all the boundary conditions stated in Equation (2.9).

So through the generalizing concept of HAM the socalled zero-order deformation equation can be written as:

$$
(1-q) L\left[\varphi(x, q)-u_{0}(x)\right]=q h H(x) N[\varphi(x, q)]
$$

where $h \neq 0$ is the non-zero auxiliary parameter, $H(x)$ is the auxiliary function, and $L$ is the auxiliary linear operator which is chosen here as

$$
\mathcal{L}(f(x))=\frac{\mathrm{d}^{2} f(x)}{\mathrm{d} x^{2}}
$$

with the following property:

$$
\mathcal{L}\left(C_{1} x+C_{2}\right)=0 \text { when } C_{1} x+C_{2}=0
$$

Expanding $\varphi(x, q)$ in Taylor series with respect to the embedding parameter $q$, one obtains

$$
\begin{aligned}
& \varphi[x, q]=u_{0}(x)+\sum_{m=1}^{\infty} u_{m}(x) q^{m} \\
& u_{m}(x)=\left.\frac{1}{m !} \frac{\partial^{m} \varphi[x, q]}{\partial q^{m}}\right|_{q=0}
\end{aligned}
$$

With due attention to the procedure of HAM [21], $u_{m}(x)$ should be chosen so as the following equation is satisfied

$$
\left.\frac{\mathrm{d} u_{m}}{\mathrm{~d} X}\right|_{X=0}=u_{m}(1)=0
$$

If the series $\varphi(x, q)$ converges at $q=1$, then the series solution is

$$
\varphi[x, 1]=u_{0}(x)+\sum_{m=1}^{\infty} u_{m}(x)
$$

where $u_{m}(x)$ could be obtained by the so-called highorder deformation equation. For obtaining the $m^{\text {th }}$-order deformation equation, the following vector is defined as:

$$
\boldsymbol{u}_{n}=\left\{u_{0}(x), u_{1}(x), \cdots, u_{n}(x)\right\}
$$

Differentiating both sides of the zero-order equation $m$ times with respect to $q$ and then setting $q=0$, the socalled $m^{\text {th }}$-order deformation equation can be obtained as

$$
L\left[u_{m}(x)-\chi_{m} u_{m-1}(x)\right]=h H(x) R_{m}\left(\boldsymbol{u}_{m-1}, x\right)
$$

where

$$
\begin{gathered}
\chi_{m}=\left\{\begin{array}{lc}
0, & m \leq 1 \\
1, & \text { otherwise }
\end{array}\right. \\
R_{m}\left(\boldsymbol{u}_{m-1}, x\right)=\left.\frac{1}{(m-1) !} \frac{\partial^{m-1} N[\varphi(x, q)]}{\partial q^{m-1}}\right|_{q=0}
\end{gathered}
$$

Therefore, the following relation is obtained

$$
\begin{gathered}
R_{m}\left(\boldsymbol{u}_{m-1}, x\right)=u_{m-1}^{\prime \prime}+\alpha \sum_{i=0}^{m-1} u_{i} u_{m-1-i}^{\prime \prime} \\
+\beta \sum_{i=0}^{m-1} u_{m-1-i} \sum_{k=0}^{i} u_{k} u_{i-k}^{\prime \prime}-K u_{m-1}
\end{gathered}
$$

We are free to choose the auxiliary parameter $h$, the auxiliary function $H(x)$, the initial guess $u_{0}(x)$, and the auxiliary linear operator $L$ so that the validity and flexibility of the HAM solution to control the convergence region is proven. Due to the rule of solution expression [21] the auxiliary function is chosen as follows

$$
H(x)=1
$$

According to the HAM, the valid region of the auxil- 
iary parameter $h$ for convergence of the solution series is the flat regions of $h$-curves.

\section{Results and Discussion}

To see the proper values of $h$, the $h$-curves are plotted for different values of dimensionless parameters

$\alpha, \beta$ and $K$ in Figure 1 to obtain the valid results of the considered conditions.
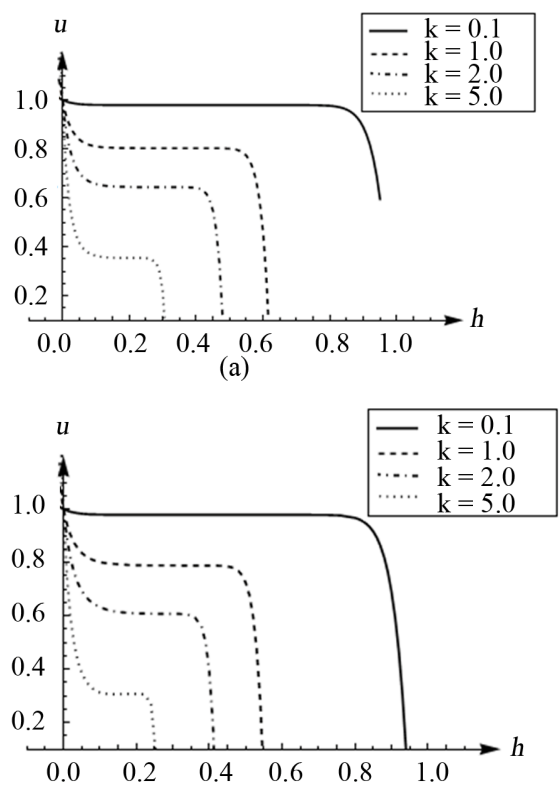

(b)

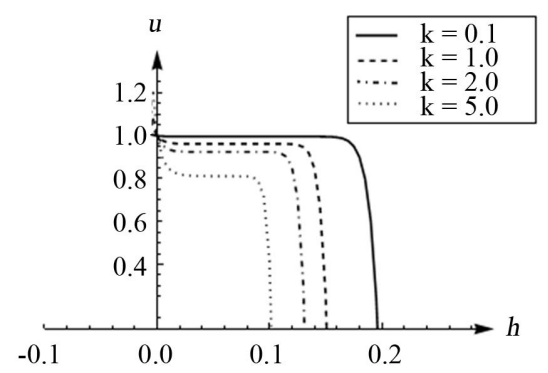

(c)

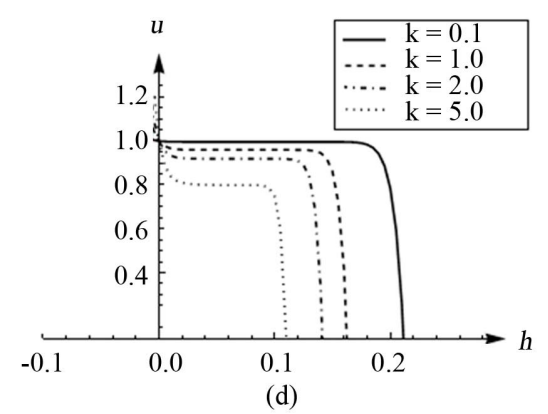

Figure 1. Variations of $u(x)$ versus non-dimensional paramete $x$ for (a) $\alpha=1.0, \beta=0.1$; (b) $\alpha=0.1, \beta=1.0$; (c) $\alpha=10.0, \beta=0.1$; and (d) $\alpha=10.0, \beta=1.0$.
The procedure of solving the non-dimensional equation of enzyme reaction (Equation (2.8)) which is based on the non-Michaelis-Menten kinetics theory utilizing HAM is described in Section 3. It is mentioned that $m^{\text {th }}$-order deformation equation should be employed to solve the problem. As the first step of the solution, the diagrams of variation of non-dimensional parameter $u(x)$ versus auxiliary parameter $h$ for different investigated cases are illustrated (Figure 1). Then, flat regions of $h$-curves are obtained employing these diagrams.

On the basis of the chosen values of auxiliary parameter $h$ in the flat regions of $h$-curves (Figure 1) the variations of $u(x)$ versus $x$ were examined (Figure 2) to clarify the dependency of these variations on different non-dimensional parameters defined in Equation (2.8). Figure 2 clearly demonstrates that the effect of variation of non-dimensional parameter $K$ on the profiles of $u(x)$ is so important which causes large differences between values of $u(x)$ for different values of $K$. Values of $u(x)$ at different locations are presented in Table $\mathbf{1}$ and Table 2 for better clarifying the effects of $K$ as well as other non-dimensional parameters $(\alpha, \beta)$.

Table 1. Values of non-dimensional variable $u(x)$ at different locations for $\alpha=1.0, \beta=0.1, \alpha=0.1, \beta=1.0$ and for different values of non-dimensional parameter $K$.

\begin{tabular}{cccccccccc}
\hline$x$ & \multicolumn{3}{c}{$\alpha=1.0, \beta=0.1$} & \multicolumn{3}{c}{$\alpha=0.1, \beta=1.0$} \\
\hline & $K=0.1$ & $K=1.0$ & $K=2.0$ & $K=5.0$ & $K=0.1$ & $K=1.0$ & $K=2.0$ & $K=5.0$ \\
\hline 0 & 0.9764 & 0.7831 & 0.6095 & 0.3012 & 0.9762 & 0.7675 & 0.5697 & 0.2517 \\
0.2 & 0.9773 & 0.7916 & 0.6264 & 0.3245 & 0.9771 & 0.7767 & 0.5862 & 0.2517 \\
0.4 & 0.9802 & 0.8172 & 0.6695 & 0.3971 & 0.9800 & 0.8044 & 0.6362 & 0.3515 \\
0.6 & 0.9849 & 0.8601 & 0.7458 & 0.5261 & 0.9848 & 0.8507 & 0.7209 & 0.4885 \\
0.8 & 0.9915 & 0.9209 & 0.8551 & 0.7225 & 0.9914 & 0.9159 & 0.8417 & 0.7002 \\
1.0 & 1.0000 & 1.0000 & 1.0000 & 1.0000 & 1.0000 & 1.0000 & 1.0000 & 1.0000 \\
\hline
\end{tabular}

Table 2. Values of non-dimensional variable $u(x)$ at diferent locations for $\alpha=10.0, \beta=0.1, \alpha=10.0, \beta=1.0$ and for different values of non-dimensional parameter $K$.

\begin{tabular}{cccccccccc}
\hline$x$ & \multicolumn{3}{c}{$\alpha=10.0, \beta=0.1$} & & $\alpha=10.0, \beta=1.0$ \\
\hline & $K=0.1$ & $K=1.0$ & $K=2.0$ & $K=5.0$ & $K=0.1$ & $K=1.0$ & $K=2.0$ & $K=5.0$ \\
\hline 0 & 0.9955 & 0.9551 & 0.9105 & 0.7795 & 0.9958 & 0.9583 & 0.9167 & 0.7927 \\
0.2 & 0.9957 & 0.9569 & 0.9141 & 0.7882 & 0.9960 & 0.9600 & 0.9200 & 0.8010 \\
0.4 & 0.9962 & 0.9623 & 0.9248 & 0.8146 & 0.9965 & 0.9650 & 0.9300 & 0.8258 \\
0.6 & 0.9971 & 0.9712 & 0.9427 & 0.8586 & 0.9973 & 0.9733 & 0.9467 & 0.8672 \\
0.8 & 0.9984 & 0.9838 & 0.9678 & 0.9204 & 0.9985 & 0.9850 & 0.9700 & 0.9253 \\
1.0 & 1.0000 & 1.0000 & 1.0000 & 1.0000 & 1.0000 & 1.0000 & 1.0000 & 1.0000 \\
\hline
\end{tabular}




\section{Verification of the Solution}

In order to verify the accuracy of the obtained solution, results are compared with available simulation and exact solutions available in the literature [28] for the special case of $\alpha=0, \beta=0$ (Table 3 ). It is shown that the values of the results of HAM and exact solution in the considered special case are identical to each other at the considered numerical precision. It should be noted that exact solutions are available only for this special case. The excellent agreements between HAM and exact solutions in Table 3 suggest that HAM can yield highly accurate solutions not only for the special cases but also for the general cases for which exact solution does not exist. Hence, the results presented in this paper can be utilized as promising data for investigating the behavior of the general enzyme reaction.

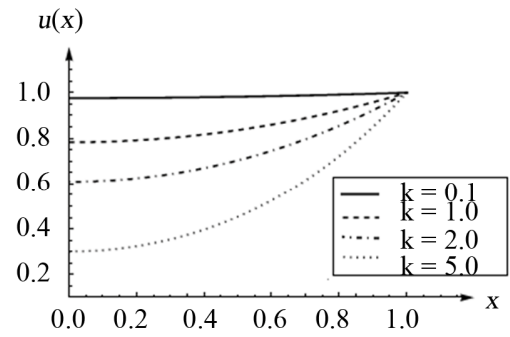

(a)

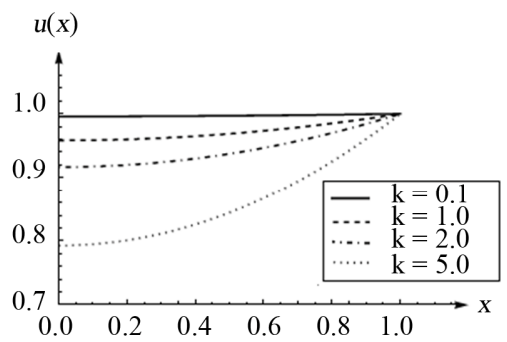

(c)

\section{Conclusion}

Analytical solution of the amperometric biosensor at mixed enzyme kinetics and diffusion limitation is presented utilizing HAM. Dimensionless equation of the problem is obtained using the mathematical modeling presented in the paper which is based on non-Michaelis-Menten kinetics of the enzymatic reaction. Solution procedure of the non-dimensional equation of enzyme reaction is described and $m^{\text {th }}$-order deformation equation is obtained on the basis of the non-dimensional enzyme reaction equation presented in this article. Several $h$-curves are presented to show the convergence region of the solution. Results of the solution are presented for different quantities of the dimensionless parameters used to non-dimensionalized the enzyme reaction equation. It is clarified that the most effective parameter in the reac-

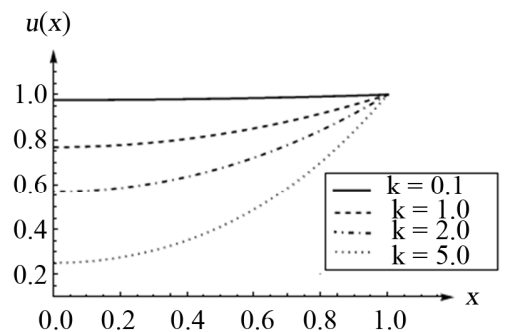

(b)

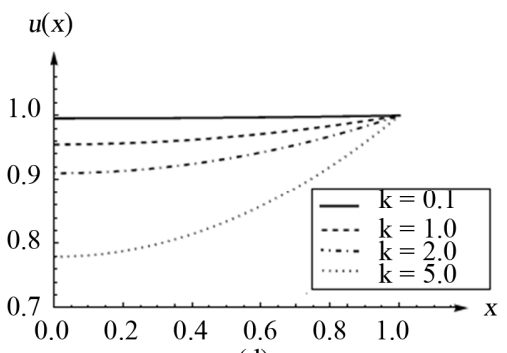

(d)

Figure 2. Variations of $u(x)$ versus auxiliary parameter $x$ for (a) $\alpha=1.0, \beta=0.1 ;$ (b) $\alpha=0.1, \beta=1.0 ;$ (c) $\alpha=10.0, \beta=0.1$; and (d) $\alpha=10.0, \beta=1.0$.

Table 3. Comparison of the results of the HAM with simulation and actual results of the problem at different locations and for different values of non-dimensional parameter $K(\alpha=0, \beta=0)$.

\begin{tabular}{|c|c|c|c|c|c|c|c|c|c|}
\hline & & $K=0.1$ & & & $K=1.0$ & & & $K=5.0$ & \\
\hline $\mathrm{X}$ & Simulation & HAM & Exact & Simulation & HAM & Actual & Simulation & HAM & Actual \\
\hline 0 & 0.9500 & 0.9520 & 0.9520 & 0.6500 & 0.6481 & 0.6481 & 0.2100 & 0.2113 & 0.2113 \\
\hline 0.25 & 0.9529 & 0.9550 & 0.9550 & 0.6666 & 0.6684 & 0.6684 & 0.2502 & 0.2452 & 0.2452 \\
\hline 0.50 & 0.9613 & 0.9639 & 0.9639 & 0.7293 & 0.7303 & 0.7303 & 0.3585 & 0.3578 & 0.3578 \\
\hline 0.75 & 0.9767 & 0.9789 & 0.9789 & 0.8366 & 0.8390 & 0.8390 & 0.5893 & 0.5851 & 0.5851 \\
\hline 1.0 & 0.9976 & 1.0000 & 1.0000 & 0.9940 & 1.0000 & 1.0000 & 0.9970 & 1.0000 & 1.0000 \\
\hline
\end{tabular}


tion and local dependency of the dependent variable of the problem $u(x)$ is $K$. Conclusively, some available results in the literature are used to prove the high accuracy of the presented solution.

\section{REFERENCES}

[1] F. Scheller and F. Schubert, "Biosensors,” Elsevier, Amsterdam, 1988.

[2] U. Wollenberger, F. Lisdat and F. W. Scheller, "Enzymatic Substrate Recycling Electrodes," Frontiers in Biosensorics II, Vol. 81, 1997, pp. 45-70.

[3] M. Pohanka, P. Skladal and M. Kroca, "Biosensors for Biological Warfare Agent Detection,” Defense Science Journal, Vol. 57, No. 3, 2007, pp. 185-193.

[4] M. Pohanka, D. Jun and K. Kuca, "Mycotoxin Assay Using Biosensor Technology: A Review," Drug and Chemical Toxicology, Vol. 30, No. 3, 2007, pp. 253-261. http://dx.doi.org/10.1080/01480540701375232

[5] S. Haron and A. K. Ray, "Optical Biodetection of Cadmium and Lead Ions in Water," Medical Engineering and Physics, Vol. 28, No. 10, 2006, pp. 978-981. http://dx.doi.org/10.1016/j.medengphy.2006.04.004

[6] A. J. Baeumner, C. Jones, C. Y. Wong and A. Price, “A Generic Sandwich-Type Biosensor with Nanomolar Detection Limits," Analytical and Bioanalytical Chemistry, Vol. 378, No. 6, 2004, pp. 1587-1593. http://dx.doi.org/10.1007/s00216-003-2466-0

[7] K. R. Rogers, "Biosensors for Environmental Applications,” Biosensors and Bioelectronics, Vol. 10, No. 6-7, 1995, pp. 533-541. http://dx.doi.org/10.1016/0956-5663(95)96929-S

[8] A. P. F. Turner, I. Karube and G. S. Wilson, "Biosensors: Fundamentals and Applications," Oxford University Press, Oxford, 1987.

[9] U. Wollenberger, F. Lisdat and F. W. Scheller, "Frontiers in Biosensorics 2. Practical Applications," Birkhauser Verlag, Basel, 1997.

[10] A. Chaubey and B. D. Malhotra, "Mediated Biosensors," Biosensors and Bioelectronics, Vol. 17, No. 6-7, 2002, pp. 441-456. http://dx.doi.org/10.1016/S0956-5663(01)00313-X

[11] G. G. Guilbault and G. Nagy, “An Improved Urea Electrode," Analytical Chemistry, Vol. 45, No. 2, 1973, pp. 417-419. http://dx.doi.org/10.1021/ac60324a053

[12] L. D. Mell and J. T. Maloy, "A Model for the Amperometric Enzyme Electrode Obtained through Digital Simulation and Applied to the Glucose Oxidase System," Analytical Chemistry, Vol. 47, No. 2, 1975, pp. 299-307. http://dx.doi.org/10.1021/ac60352a006

[13] J. D. Hoffman, "Numerical Methods for Engineers and Scientists,” McGraw-Hill, New York, 1992.

[14] T. Schulmeister, "Mathematical Modeling of the Dynamic Behavior of Ampero-Metric Enzyme Electrodes," Selective Electrode Reviews, Vol. 12, 1990, pp. 203-260.

[15] R. Aris, “The Mathematical Theory of Diffusion and Re- action in Permeable Catalysts: The Theory of the Steady State,” Clarendon Press, Oxford, 1975.

[16] L. K. Bieniasz and D. Britz, "Recent Developments in Digital Simulation of Electroan-Alytical Experiments," Polish Journal of Chemistry, Vol. 78, 2004, pp. 11951219.

[17] S. J. Liao, "Beyond Perturbation: Introduction to the Homotopy Analysis Method," Chapman and Hall/CRC Press, Boca Raton, 2003. http://dx.doi.org/10.1201/9780203491164

[18] A. H. Nayfeh, "Problems in Perturbation," 2nd Edition, Wiley, New York, 1993.

[19] S. J. Liao, “The Proposed Homotopy Analysis Technique for the Solution of Nonlinear Problems,” Ph.D. Thesis, Shanghai Jiao Tong University, Shanghai, 1992.

[20] P. Manimozhi, A. Subbiah and L. Rajendran, "Solution of Steady-State Substrate Concentration in the Action of Biosensor Response at Mixed Enzyme Kinetics," Sensors and Actuators B, Vol. 147, No. 1, 2010, pp. 290-297. http://dx.doi.org/10.1016/j.snb.2010.03.008

[21] S. Abbasbandy, T. Hayat, "Solution of the MHD FalknerSkan Flow by Homotopy Analysis Method," Communications in Nonlinear Science and Numerical, Vol. 14, No. 9-10, 2009, pp. 3591-3598. http://dx.doi.org/10.1016/j.cnsns.2009.01.030

[22] Y. M. Chen and J. K. Liu, “A Study of Homotopy Analysis Method for Limit Cycle of Van Der Pol Equation," Communications in Nonlinear Science and Numerical, Vol. 14, No. 5, 2009, pp. 1816-1821. http://dx.doi.org/10.1016/j.cnsns.2008.07.010

[23] G. Craciun, J. W. Helton and R. J. Williams, "Homotopy Methods for Counting Reaction Network Equilibria," Mathematical Biosciences, Vol. 216, No. 2, 2008, pp. 140-149. http://dx.doi.org/10.1016/j.mbs.2008.09.001

[24] M. S. H. Chowdhury, T. H. Hassan and S. Mawa, "A New Application of Homotopy Perturbation Method to the Reaction-diffusion Brusselator Model," ProcediaSocial and Behavioral Sciences, Vol. 8, 2010, pp. 648653. http://dx.doi.org/10.1016/j.sbspro.2010.12.090

[25] D. M. Dunlavy and C. Prospectus, “A Homotopy Method for Predicting the Lowest Energy Conformations of Proteins,” 18 April 2003.

[26] R. Baronas, F. Ivanauskas, J. Kulys and M. Sapagovas, "Modeling of Amperometri Biosensors with Rough Surface of the Enzyme Membrane," Journal of Mathematical Chemistry, Vol. 34, No. 3-4, 2003, pp. 227-242. http://dx.doi.org/10.1023/B:JOMC.0000004072.97338.12

[27] C. V. Pao, "Mathematical Analysis of Enzyme-Substrate Reaction Diffusion Insomebiochemical Systems," Nonlinear Analysis: Theory, Methods \& Applications, Vol. 4, No. 2, 1979, pp. 369-392. http://dx.doi.org/10.1016/0362-546X(80)90061-9

[28] P. Manimozhi, A. Subbiah and L. Rajendran, "Solution of Steady-State Substrate Concentration in the Action of Biosensor Response at Mixed Enzyme Kinetics,” Sensors and Actuators B, Vol. 147, 2010, pp. 290-297. http://dx.doi.org/10.1016/j.snb.2010.03.008 